\title{
On the vibration of a cracked rotating blade
}

\author{
Ming-Chuan Wu and Shyh-Chin Huang * \\ Department of Mechanical Engineering, National \\ Taiwan University of Science and Technology, \\ 43 Keelung Road, Sec. 4, Taipei, Taiwan 106, ROC
}

Received 2 March 1996

Revised 22 October 1998

The dynamic behavior of a rotating blade containing a transverse crack was investigated. First, the local flexibility of the cracked blade was obtained by using the method of the released energy. An energy principle, in conjunction with a weighted residual method, was then applied to yield the discrete equations of motion. The equations of motion were further utilized to study the influences of the crack depth and location on the bending natural frequencies under various of rotation speeds. The numerical calculation showed that the crack effects the natural frequencies and the response appreciably only if it is relatively deep and locates near the root of the blade. However, the effects increase exponentially with the depth increases. In addition to the natural frequencies, the displacement responses of the blade with a crack under a constant lateral forces were discussed as well. This was done by calculating the deflections at the tip of the blade for various crack depths and locations. Similar to the rotation speed of the blade frequency, the deflection was offset by the increase of the rotation. However, the centrifugal effects increased significantly such that the crack's effects became relatively insignificant. Nevertheless, the study showed that the changes on the natural frequency and the tip-deflection of the blade due to a crack may be used as indices for on-line detection of cracks.

Keywords: Crack, vibration

\section{Introduction}

Due to manufacturing flaws or cyclic fatigue during operation, cracks frequently appear in rotating machinery. Cracks in a structural member may be diagnosed off-line by many methods, such as the methods of ultrasonic detection and acoustic emission. A crack causes local flexibility which, more or less, changes the

\footnotetext{
*Corresponding author. Tel.: +11 8862 27376443; Fax: +11 886 2 27376460; E-mail: huang@vib2.me.ntust.edu.tw.
}

dynamic behaviors of structure. On the other hand, online detection, usually utilizing vibration signal analysis, offers possibilities of early crack knowledge and remaining life prediction for damaged structures. Thus, to develop a reliable on-line detection, thorough understanding of crack behavior and its effects to structure vibration is necessary. As the consequence, investigators have been continuously working on the mathematical modeling and vibration analyses of cracks for years.

For example, Kieshmer [10] and Thomson [22], based on an equivalent reduced section, studied the influence of cracks on the dynamic response of a damaged beam, and illustrated the effect of the crack depth and the crack location to the natural frequencies and vibration modes. Gasch [8] developed a hinge model for a DeLaval rotor, in which additional crack flexibility was introduced and the crack was switched on/off by a step function. His research concluded that there exists great similarity between a cracked shaft and an unequal cross-sectional shaft.

Mayes and Davies [11,12] employed the Green function to calculate the compliance of a cracked shaft. In a later paper [13], they replaced the crack by a localized, reduced-diameter shaft. Grabowski [9] utilized the modal formulation to derive an analytical model and obtained a set of discrete, periodic time-varying coefficients equations for a cracked shaft.

Nelson and Nataraj [14] used a switching function, according to shaft's curvature at crack location, for crack's open/close and solved for the dynamic response with a finite element method. Christides and Barr [5] assumed an exponential stress distribution in the vicinity of the crack, and evaluated the exponent experimentally. However, the exponential approximation was yet valid for notches only.

Chondros and Dimarogonas [4] investigated the relation between the change in natural frequency of vibration of a cantilever beam and the crack depth that appears at the built-in edge which is clamped by way of a weld. Dimarogonas and Paipetis [6], from the theories of fracture mechanics, constructed a crack related $5 \times 5$ local flexibility matrix for a rectangular beam and analyzed the coupling effects between the axial load- 
ing and lateral bending. In another paper, Dimarogonas and Papadopoulos [7] expanded the work to a circular shaft with a transverse crack, and calculated the flexibility coefficients due to bending in two perpendicular directions. In their continuous papers [16-19], they introduced the complete $6 \times 6$ local flexibility matrix for a transverse surface crack on a shaft. Finally, they derived the cross-couplings of longitudinal, bending, and torsional vibration and developed a spectral method for crack identification.

Rizos et al. [20] illustrated the identification of the crack in a cantilever beam from the measured amplitudes of the two points of structure that vibrating at one of its natural modes. Wu and Huang [24] displayed a feasible technique, achieved by intersecting the two equi-amplitude response curves from two separated sensing probes, to identify the crack depth and crack location on a rotating shaft-disk. Chen and Chen [2] employed a finite element model to investigate the dynamic instability effects of a crack on a thick rotating blade. A later paper [3] focused on the pretwisted angle of the rotating blade. Ostachowicz and Krawczuk [15] analyzed the effect of two open cracks (double-sided and single-sided) upon the frequencies of the natural flexural vibrations in a cantilever beam. Adams et al. [1] evaluated the integrity of structures by vibration technique, the axial modes of vibration were used to predict the actual damage sites of the bars.

In the present paper, a rotating blade with a transverse crack is investigated. The local flexibility coefficients given by Dimarogonas and Paipetis [6] are first adopted, then an energy approach is applied for evaluating the energy change of a rotating cracked blade. Extended Hamilton's principle in conjunction with a weighted residual method follows to yield the discrete equations of motion. The effects of crack size, crack location and rotating speed on the first few natural frequencies are discussed. The displacement of the cracked blade due to lateral forces is also estimated. Particularly, the increment of tip end due to root crack is discussed.

\section{Energy form}

A uniform blade containing a transverse crack is shown in Fig. 1, where one end of the blade is clamped onto a rotating hub of radius $r$. Coordinates set $X Y$ is the inertia frame and $x y$, fixed with the hub, is the rotating set at a constant speed $\Omega$. In the following derivation, the rotating blade is considered as an

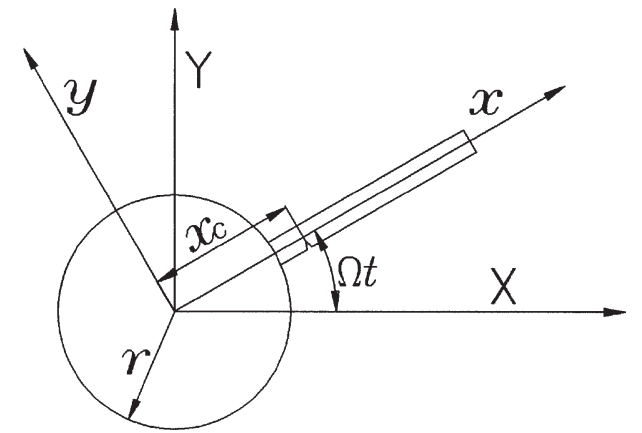

Fig. 1. A rotating blade containing a transverse crack.

Euler's beam with a constant cross section, and only the transverse bending deflection $v(x, t)$ is concerned. First, the kinetic energy of the rotating blade is

$T=\frac{1}{2} \rho A \int_{r}^{r+L}\left[\dot{v}^{2}+\left(x^{2}+v^{2}\right) \Omega^{2}+2 x \dot{v} \Omega\right] \mathrm{d} x$,

where the upper dot denotes the differentiations with respect to time. $\rho, A, E, I, r$ and $L$, respectively, denote the density, cross-section area, Young's modulus, area moment of inertia, radius of the hub, and the length of blade. The potential energy is

$$
\begin{aligned}
U= & U^{\mathrm{e}}+U^{\mathrm{i}} \\
= & \frac{1}{2} E I \int_{r}^{r+L} v^{\prime \prime 2} \mathrm{~d} x \\
& +\frac{1}{4} \rho A \int_{r}^{r+L} \Omega^{2}\left[(r+L)^{2}-x^{2}\right] v^{\prime 2} \mathrm{~d} x,
\end{aligned}
$$

where the primes denote the differentiations with respect to displacement $x$. $U^{\mathrm{e}}$ in Eq. (2) is the energy due to bending, $U^{\mathrm{i}}$ is that due to centrifugal force, and $\frac{1}{2} \rho \Omega^{2}\left[(r+L)^{2}-x^{2}\right]$ the initial stress. The work due to lateral external force $F(x, t)$ acting on the blade is

$$
W=\int_{r}^{r+L} F(x, t) v(x, t) \mathrm{d} x .
$$

Dimarogonas and Paipetis [6] employed the Castigliano's theorem and formulated a $5 \times 5$ local flexibility matrix for a rectangular beam containing a transverse crack. According to their investigation, the coupling terms could be neglected provided the bending moment was the dominant term. The released energy of the crack was hence given as

$$
U_{\mathrm{c}}=b \int_{0}^{a} J(a) \mathrm{d} a,
$$




$$
\begin{aligned}
J(a) & =\frac{1-\nu^{2}}{E} K_{\mathrm{I} 5}^{2}, \\
K_{\mathrm{I} 5} & =\frac{6 P_{5}}{b h^{2}} \sqrt{\pi a} F_{2}\left(\frac{a}{h}\right), \\
F_{2}\left(\frac{a}{h}\right)= & \sqrt{\frac{2 h}{\pi a} \tan \left(\frac{\pi a}{2 h}\right)} \\
& \times \frac{0.923+0.199\left[1-\sin \left(\frac{\pi a}{2 h}\right)\right]^{4}}{\cos \left(\frac{\pi a}{2 h}\right)},
\end{aligned}
$$

where $J(a), K_{\mathrm{I} 5}$, and $F_{2}(a / h)$ denote the energy release rate, the stress intensity factor, and the geometric parameter of the crack, respectively. $a$ is the crack depth, $b$ and $h$ are the width and height of the blade. $P_{5}$ denote the bending moment at the crack position.

The bending moment at the crack $\left(x_{\mathrm{c}}\right)$ can be represented by $\left.E I\left(\partial^{2} v / \partial x^{2}\right)^{2}\right|_{x=x_{c}}$, as the results, the released energy $U_{\mathrm{c}}$ of the crack is

$$
\begin{aligned}
U_{\mathrm{c}} & =\left.3 E I\left(1-\nu^{2}\right) h \bar{C}_{55}\left(\frac{\partial^{2} v}{\partial x^{2}}\right)^{2}\right|_{x=x_{\mathrm{c}}} \\
& =\int_{r}^{r+L} 3 E I\left(1-\nu^{2}\right) h \bar{C}_{55} \delta^{\star}\left(x-x_{\mathrm{c}}\right) v^{\prime \prime 2} \mathrm{~d} x \\
\bar{C}_{55} & =\int_{0}^{a / h} \pi\left(\frac{a}{h}\right) F_{2}^{2}\left(\frac{a}{h}\right) \mathrm{d}\left(\frac{a}{h}\right)
\end{aligned}
$$

where $\delta^{\star}$ is the Dirac delta function, $\bar{C}_{55}$ the dimensionless crack flexibility [6].

\section{Equations of motion}

With the energy expressions in Eqs (1)-(3) and (8), the extended Hamilton's principle

$$
\delta \int_{t 1}^{t 2}\left[T-U+U_{\mathrm{c}}+W\right] \mathrm{d} t=0
$$

yields the equation of motion of the cracked blade,

$$
\begin{aligned}
& \rho A\left\{\ddot{v}-\Omega^{2} v-\frac{1}{2} \Omega^{2}\left[(r+L)^{2}-x^{2}\right] v^{\prime \prime}+\Omega^{2} x v^{\prime}\right\} \\
& +E I v^{\prime \prime \prime \prime}-6 E I\left(1-\nu^{2}\right) h \bar{C}_{55}\left[\delta^{\star}\left(x-x_{\mathrm{c}}\right) v^{\prime \prime \prime \prime}\right. \\
& \left.+2 \delta^{\star^{\prime}}\left(x-x_{\mathrm{c}}\right) v^{\prime \prime \prime}+\delta^{\star^{\prime \prime}}\left(x-x_{\mathrm{c}}\right) v^{\prime \prime}\right]=F(x, t),
\end{aligned}
$$

and the boundary conditions,

$$
\begin{array}{r}
\left.\left\{E I v^{\prime \prime}\right\} \delta v^{\prime}\right|_{x=r}=0, \\
\left.\left\{-E I v^{\prime \prime}\right\} \delta v^{\prime}\right|_{x=r+L}=0, \\
\left.\left\{-E I v^{\prime \prime \prime}+\frac{1}{2} \rho A \Omega^{2}\left[(r+L)^{2}-x^{2}\right]\right\} \delta v\right|_{x=r}=0, \\
\left.\left\{E I v^{\prime \prime \prime}-\frac{1}{2} \rho A \Omega^{2}\left[(r+L)^{2}-x^{2}\right]\right\} \delta v\right|_{x=r+L}=0 .
\end{array}
$$

Attempt to obtain the closed-form solution of the above equations is impractical, if not impossible. Thus, extended Galerkin's method is employed for the approximate solutions. The blade deformation approximated by

$$
v(x, t)=\sum_{i=1}^{n} q_{i}(t) V_{i}(x),
$$

where $V_{i}(x)$ is the $i$-th normalized mode of a nonrotating clamped-free beam, $q_{i}(t)$ the associated participation factor, or the so-called generalized coordinates, and $n$ the number of functions deemed necessary for satisfactory convergence. By enforcing the weighted residual to zero, a discretized equation of motion in matrix form is obtained,

$$
[\mathbf{M}]\{\ddot{\mathbf{q}}\}+[\mathbf{K}]\{\mathbf{q}\}=\{\mathbf{f}\},
$$

in which $[\mathbf{M}]$ is the mass matrix with entities

$$
\begin{aligned}
M_{i j}= & \int_{r}^{r+L} \rho A V_{i}(x) V_{j}(x) \mathrm{d} x=\delta_{i j}^{\star}, \\
{[\mathbf{K}]=} & {\left[\mathbf{K}^{\mathrm{e}}\right]+\left[\mathbf{K}^{\Omega}\right]-\left[\mathbf{K}^{\mathrm{c}}\right], } \\
K_{i j}^{\mathrm{e}}= & \int_{r}^{r+L} E I V_{i}^{\prime \prime}(x) V_{j}^{\prime \prime}(x) \mathrm{d} x=\omega_{\mathrm{b} i}^{2} \delta_{i j}^{\star}, \\
K_{i j}^{\Omega}= & \int_{r}^{r+L} \rho A \Omega^{2} V_{i}(x)\left\{x V_{j}^{\prime}(x)-V_{j}(x)\right. \\
& \left.-\frac{1}{2}\left[(r+L)^{2}-x^{2}\right] V_{j}^{\prime \prime}(x)\right\} \mathrm{d} x, \\
K_{i j}^{\mathrm{c}}= & \int_{r}^{r+L} 6 E I\left(1-\nu^{2}\right) h \bar{C}_{55} \\
& \times\left\{\delta^{\star}\left(x-x_{\mathrm{c}}\right) V_{i}(x) V_{j}^{\prime \prime \prime \prime}(x)\right. \\
& +2 \delta^{\star^{\prime}}\left(x-x_{\mathrm{c}}\right) V_{i}(x) V_{j}^{\prime \prime \prime}(x) \\
& \left.+\delta^{\star^{\prime \prime}}\left(x-x_{\mathrm{c}}\right) V_{i}(x) V_{j}^{\prime \prime}(x)\right\} \mathrm{d} x
\end{aligned}
$$


Table 1

Geometric and material properties of the illustrated examples

\begin{tabular}{clcc}
\hline$E$ & Young's modulus & $2.1 \times 10^{11}$ & $\left(\mathrm{~N} / \mathrm{m}^{2}\right)$ \\
\hline$\rho$ & density & 7850 & $\left(\mathrm{~kg} / \mathrm{m}^{3}\right)$ \\
$\nu$ & Poisson's ratio & 0.3 & \\
$r$ & radius of hub & 0.2 & $(\mathrm{~m})$ \\
$L$ & length of blade & 0.2 & $(\mathrm{~m})$ \\
$b$ & width of blade & 0.03 & $(\mathrm{~m})$ \\
$h$ & height of blade & 0.004 & $(\mathrm{~m})$ \\
\hline
\end{tabular}

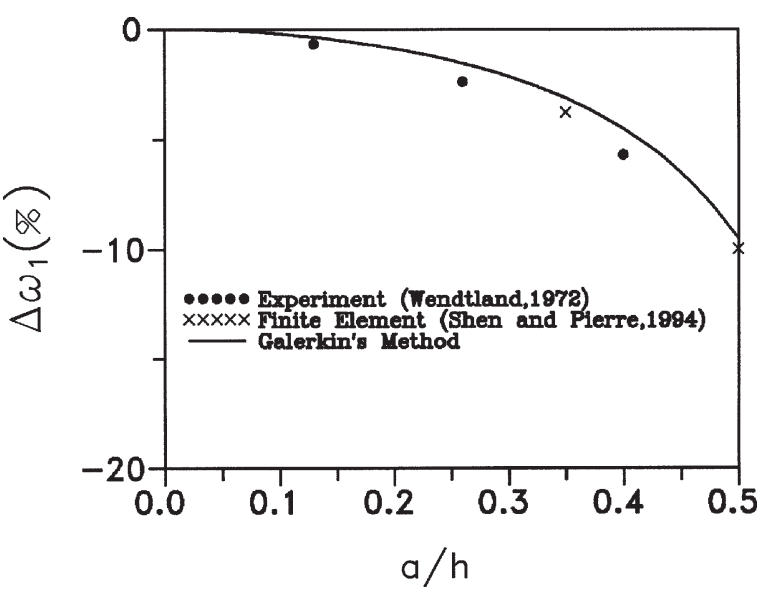

Fig. 2. Fundamental natural frequencies in terms of crack depth.

$$
\begin{aligned}
& =6 E I\left(1-\nu^{2}\right) h \bar{C}_{55} V_{i}^{\prime \prime}\left(x_{\mathrm{c}}\right) V_{j}^{\prime \prime}\left(x_{\mathrm{c}}\right), \\
f_{i} & =\int_{r}^{r+L} V_{i}(x) F(x, t) \mathrm{d} x .
\end{aligned}
$$

Note that $\omega_{\mathrm{b} i}$ is the $i$-th natural frequency of the nonrotating clamped-free beam. $\left[\mathbf{K}^{\mathrm{e}}\right],\left[\mathbf{K}^{\Omega}\right]$, and $\left[\mathbf{K}^{\mathrm{c}}\right]$ in Eq. (16) are the stiffness matrices, respectively, due to the elastic deformations, centrifugal force, and crack.

\section{Vibration analysis}

Free vibration of the rotating, cracked blade is first looked into. By omitting the forcing term in Eq. (14), i.e., let $\{\mathbf{f}\}=\{0\}$, assuming the solution in the form of

$$
\{\mathbf{q}\}=\{\mathbf{c}\} \mathrm{e}^{\mathrm{i} \omega t},
$$

and substituting Eq. (21) into Eq. (14), one obtains

$$
\left\{[\mathbf{K}]-\omega^{2}[\mathbf{M}]\right\}\{\mathbf{c}\}=\{0\} .
$$

That is, the characteristic equation of the rotating, cracked blade is then obtained as

$$
\left|[\mathbf{K}]-\omega^{2}[\mathbf{M}]\right|=0 .
$$

In order to investigate the effects of the crack, the parameters are dimensionalized as followed: $x_{\mathrm{c}}^{\star}$, defined as $\left(x_{\mathrm{c}}-r\right) / L$, is the crack location, $\omega^{\star}$ and $\Omega^{\star}$, obtained by dividing with $\frac{1}{L^{2}}(E I / \rho A)^{1 / 2}$, are the natural frequency and rotating speed, $\Delta \omega_{i}$, defined as $\left(\omega_{i}-\omega_{\mathrm{b} i}\right) / \omega_{\mathrm{b} i}$, is the $i$-th natural frequency increment (\%) of rotating cracked blade. The fundamental natural frequency of one cracked cantilever beam with $x_{\mathrm{c}}^{\star}=0.2$ and $L / 2 h=25.64$ is shown in Fig. 2. It can be seen from the figure that the Galerkin's solution (this paper) conforms to the experimental data [23] and the finite element solution [21]. As Table 1 shown, the geometric and material properties of the system are used for the following discussion.

Figs 3 and 4 showed the first four natural frequencies with $\Omega^{\star}=0$, or the non-rotating blade. It can be seen from the figures that the natural frequencies were significantly influenced by crack location. In general, the natural frequencies decreased more as the crack approached to the root of the blade except passing through some particular points.

From the above derivation, the released energy of the crack was proportional to the bending moment or the curvature at the crack. For the $n$-th mode, there are $n-1$ inflection points on nodes in the beam. Therefore, Fig. 3 showed the phenomenon that the crack did not affect a mode as it occurred at certain points. For instance, there were two interior points where the crack had no effect on the third mode frequency. The natural frequencies yet decreased rapidly as the crack approached to the root. Fig. 4 showed that, as expected, the frequencies decreased monotonically with the increase of the crack depth. The numerical results also showed that for the root crack case, $35 \%$ of crack depth $(a / h \approx 0.35)$ caused approximately $10 \%$ frequency reduction.

Fig. 5 showed the variations of the first few natural frequencies with respect to the rotating speed $\Omega^{\star}$ for $x_{\mathrm{c}}^{\star}=0$ and $a / h=0,0.4$. Each nonrotating natural frequency splits into two branches like those often seen in rotor systems. The frequency difference caused by the crack became more significant at higher modes, and the difference yet faded away with the increasing of the rotation speed.

\section{Forced vibration analysis}

Eq. (14) is now solved for forced responses. In this paper, the open crack is considered and the forces ex- 

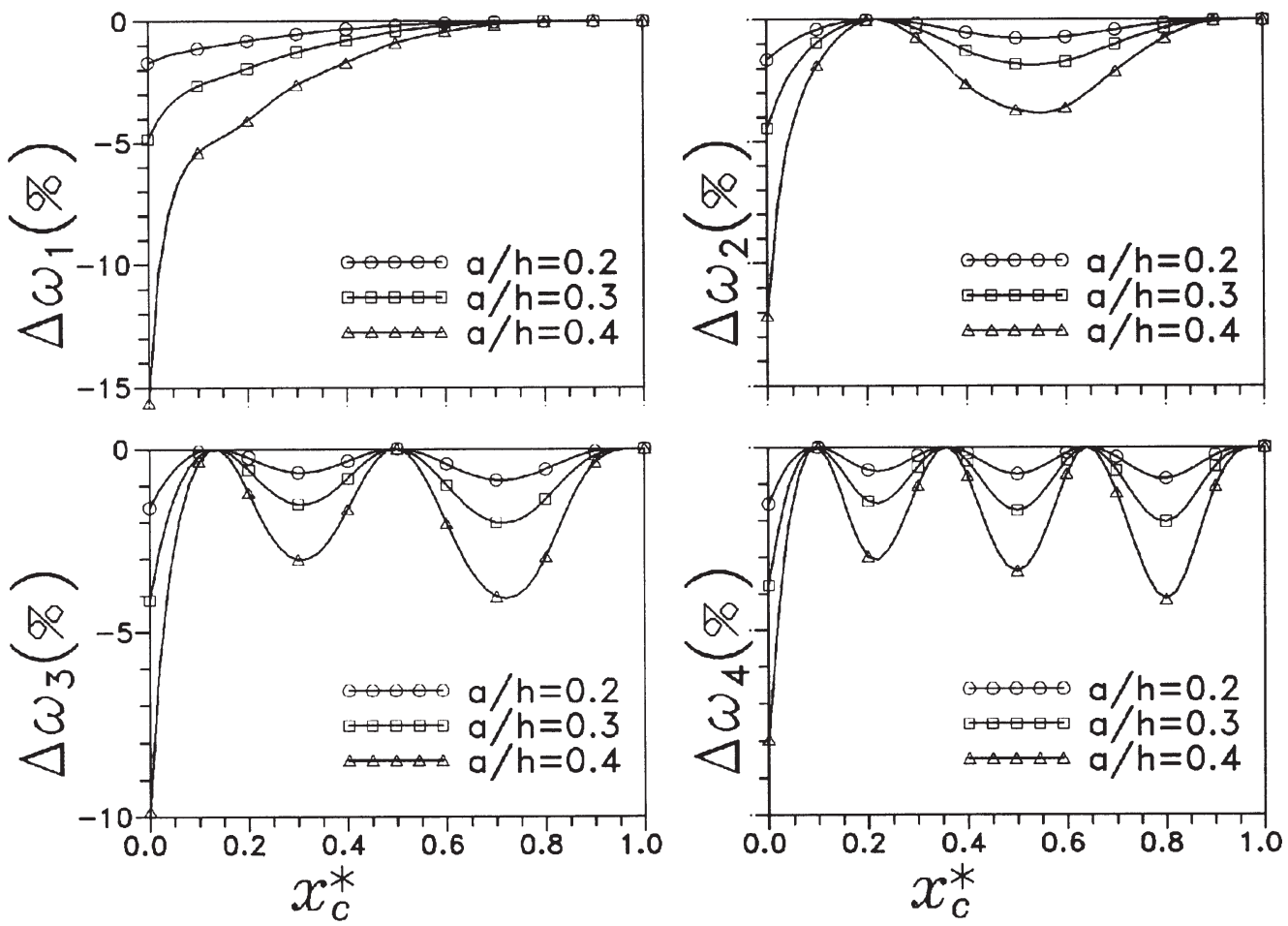

Fig. 3. Variations of natural frequencies to crack location for $\Omega^{\star}=0, a / h=0.2,0.3,0.4$.
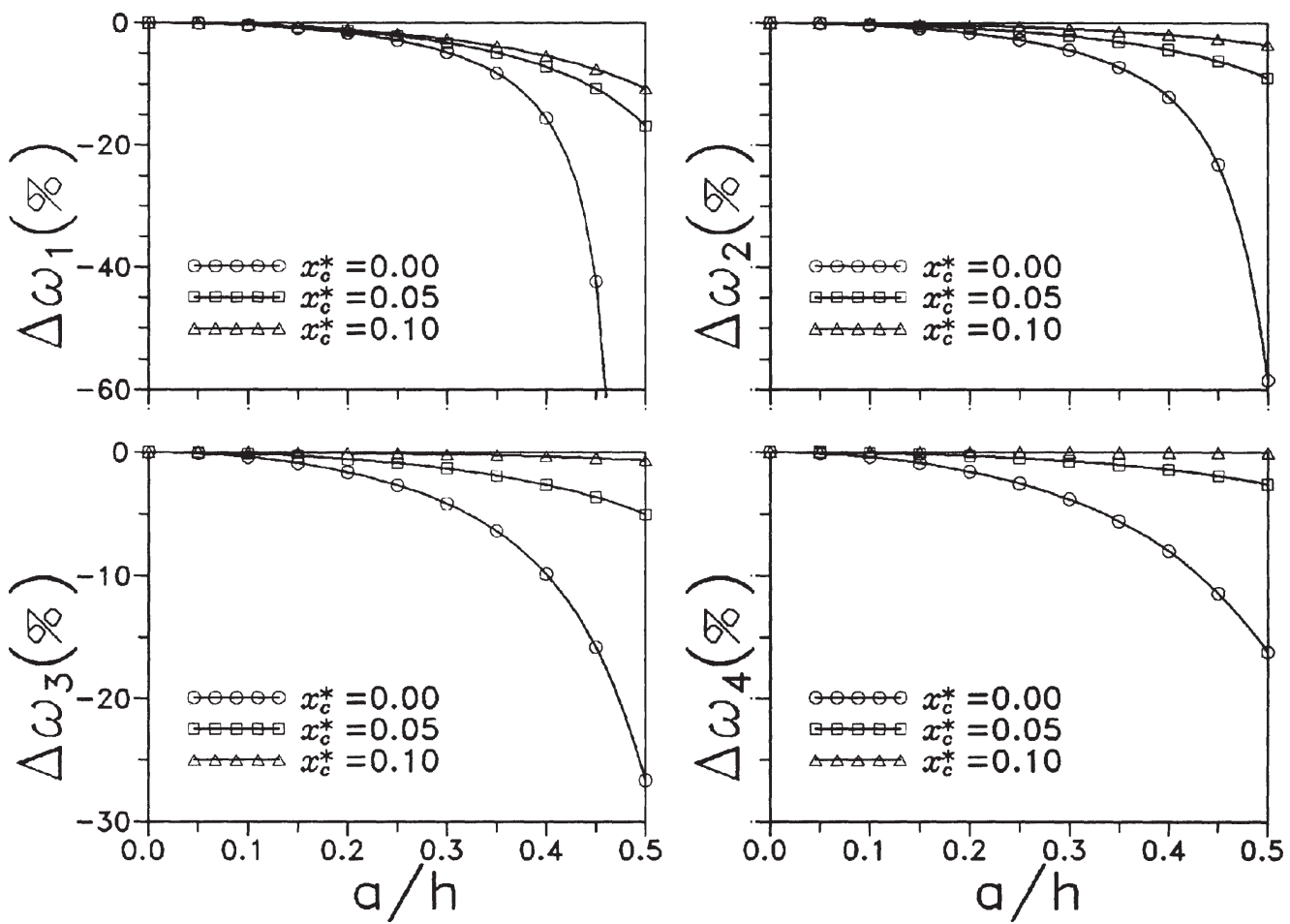

Fig. 4. Variations of natural frequencies to crack depth for $\Omega^{\star}=0, x_{\mathrm{c}}^{\star}=0,0.05,0.1$. 


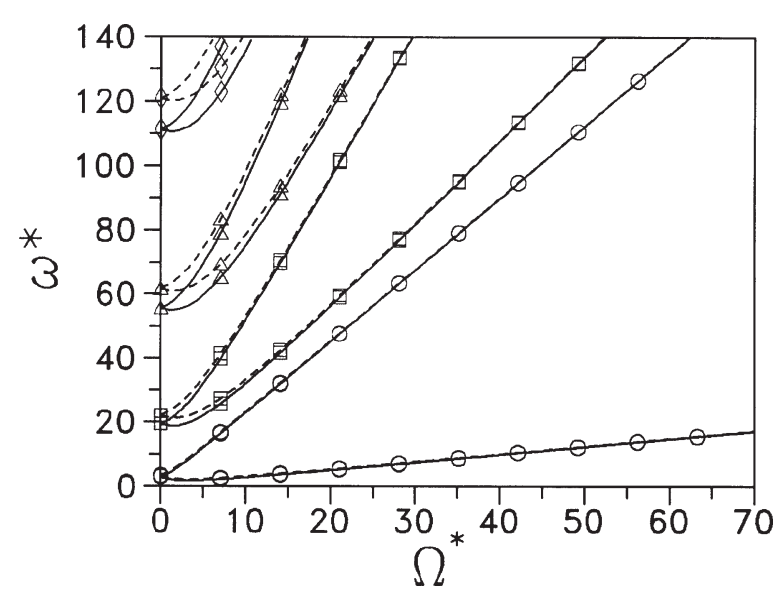

Fig. 5. Variations of natural frequencies to rotating speed for $x_{\mathrm{c}}^{\star}=0$, $a / h=0$ (dashed) and 0.4 (solid).

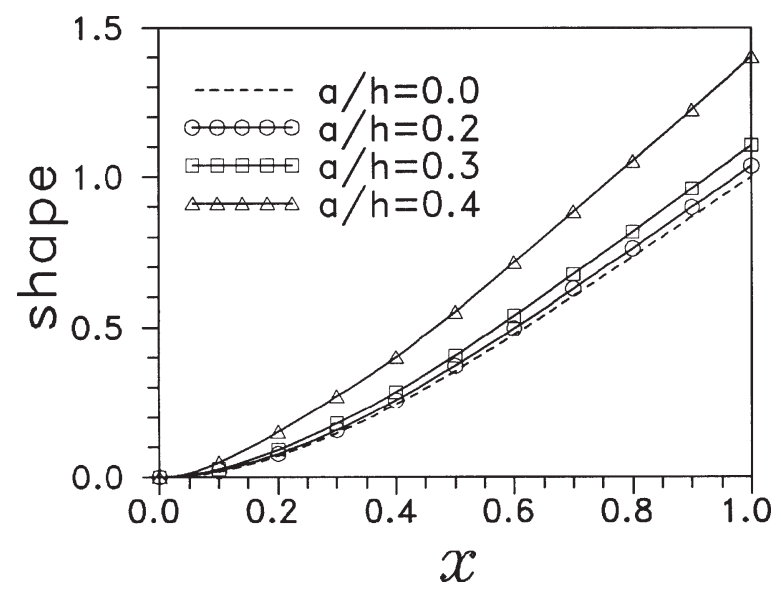

Fig. 6. Deflections of the blade for $\Omega^{\star}=0, x_{\mathrm{c}}^{\star}=0$.

erted on the blade are simplified to be constant and uniform. Then, the steady state deflection of the blade is obtained as

$$
\{\mathbf{q}\}=[\mathbf{K}]^{-1}\{\mathbf{f}\} .
$$

The deflective shapes of the nonrotating, cracked blade under such a load are shown in Fig. 6 for various cracked depth, in which the crack is located at the root. Alike than the deformation of an uncracked clampedfree beam, the cracked blade deflects more with the crack depth increases.

Let $\Delta v$ denote the increase percentage of the tip deflection due to the crack. Fig. 7 shows the variations of $\Delta v$ with crack depth and location under two rotating speed, $\Omega=0$ and $\Omega=\frac{1}{2} \omega_{\mathrm{b} 1}$. From this figure, a few phenomena were observed. Firstly, it was seen the crack effects were almost inappreciable when the

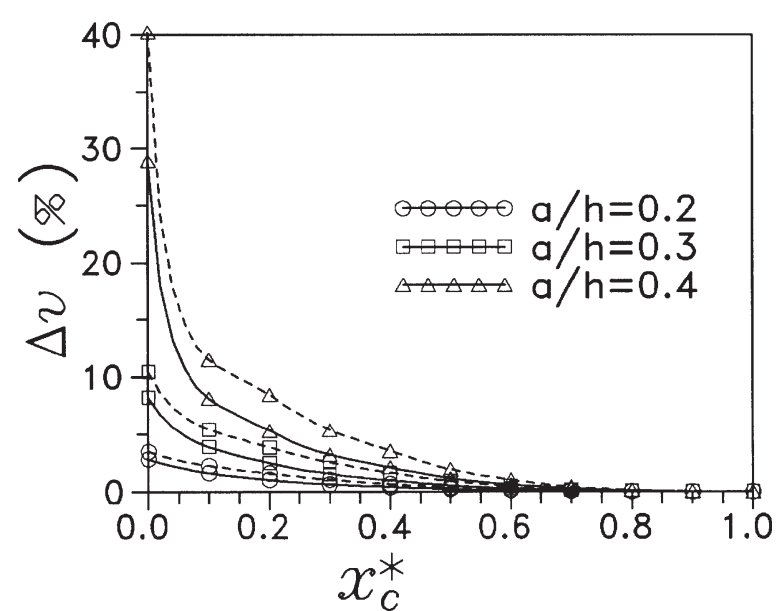

Fig. 7. Variations of tip deflection to crack depth and location for $\Omega=0$ (dashed), $\frac{1}{2} \omega_{\mathrm{b} 1}$ (solid).

crack occurred close to the tip end. Secondly, the difference of the two curves seemed to increase exponentially with the larger crack depth. This difference was also seen to decrease with increase of rotation. Centrifugal forces stiffened the blade and consequently the portion of crack effect reduced.

\section{Conclusions}

The authors formulated and investigated the problems of a rotating blade containing a transverse crack. The approach employed the crack released energy in conjunction with a weighted residual method. Numerical results were shown and some conclusions were drawn accordingly. (i) The dynamic characteristics of the rotating blade were significantly influenced by the location of the crack. (ii) The natural frequencies decreased rapidly when the crack approached to the root of the blade. However, if the crack happened at inflection points of a specific mode, the natural frequency of that mode was hardly changed. (iii) A deeper crack always imposed more natural frequencies drops, as expected. (iv) For a shallow crack, unless the crack was close to the root of the blade, the variations of natural frequencies was almost inappreciable. (v) The deflection curves of the cracked blade under constant force appeared just like the normal deformation of a cantilever beam. The tip deflection was, however, enlarged due to the crack.

Both the natural frequencies and deflective responses were affected by the crack. They were hence potential indices for crack identification. To develop a sophisticated on-line technique for crack detection, quantitative studies of those indices must be conducted. 


\section{Acknowledgement}

The work reported here was supported by the National Science Council, Taiwan, Under the grant No. NSC83-0424-E011-004.

\section{References}

[1] R.D. Adams, P. Cawley, C.J. Pye and B.J. Stone, A vibration technique for non-destructively assessing the integrity of structures, J. Mechanical Engineering Science 20 (1978), 93-100.

[2] L.W. Chen and C.L. Chen, Vibration and stability of cracked thick rotating blades, Computers and Structures 28 (1988), 6774.

[3] L.W. Chen and C.H. Jeng, Vibrational analyses of cracked pretwisted blades, Computers and Structures 46 (1993), 133-140.

[4] T.G. Chondros and A.D. Dimarogonas, Identification of cracks in welded joints of complex structures, J. Sound and Vibration 69 (1980), 531-538.

[5] S. Christides and A.D.S. Barr, One-dimensional theory of cracked Bernoulli-Euler beams, Int. J. Mechanical Sciences $\mathbf{2 6}$ (1984), 639-648.

[6] A.D. Dimarogonas and S.A. Paipetis, Analytical Methods in Rotor Dynamics, Appl. Sci., London, 1983.

[7] A.D. Dimarogonas and C.A. Papadopoulos, Vibration of cracked shafts in bending, American Society of Mechanical Engineers, Journal of Mechanical Design 102 (1983), 140-146.

[8] R. Gasch, Dynamic behavior of a simple rotor with a crosssectional crack, I Mech E Conference Publication, Vibration in Rotating Machinery, Paper No. C178/76, 1976.

[9] B. Grabowski, The vibrational behavior of a turbine rotor containing a transverse crack, ASME J. Mechanical Design 102 (1980), 140-146.

[10] P.G. Kirshmer, The effect of discontinuities on the natural frequency of beams, Proc. American Society of Testing and Materials 44 (1944), 897-904.

[11] I.W. Mayes and W.G.R. Davies, The vibrational behavior of a rotating shaft system containing a transverse crack, I Mech E Conference Publication, Vibration in Rotating Machinery, Paper No. C168/76, 1976.
[12] I.W. Mayes and W.G.R. Davies, A method of calculating the vibrational behavior of coupled rotating shaft containing a transverse crack, I Mech E Conference Publication, Vibration in Rotating Machinery, Paper No. C254/80, 1980.

[13] I.W. Mayes and W.G.R. Davies, Analysis of the response of a multi-rotor-bearing system containing a transverse crack in a rotor, ASME J. Vibration, Acoustics, Stress, and Reliability in Design 106 (1984), 139-145.

[14] H.D. Nelson and C. Nataraj, The dynamic of a rotor system with a cracked shaft, ASME J. Vibration, Acoustics, Stress, and Reliability in Design 108 (1986), 189-196.

[15] W.M. Ostachowicz and M. Krawczuk, Analysis of the effect of cracks on the natural frequencies of a cantilever beam, J. Sound and Vibration 150 (1991), 191-201.

[16] C.A. Papadopoulos and A.D. Dimarogonas, Coupled longitudinal and bending vibration of a rotating shaft with an open crack, J. Sound and Vibration 117 (1987), 81-93.

[17] C.A. Papadopoulos and A.D. Dimarogonas, Stability of cracked rotors in the coupled vibration mode, ASME J. Vibration, Acoustics, Stress, and Reliability in Design 110 (1988), 357-359.

[18] C.A. Papadopoulos and A.D. Dimarogonas, Coupled longitudinal and bending vibration of a cracked shaft, ASME J. Vibration, Acoustics, Stress, and Reliability in Design 110 (1988), $1-8$.

[19] C.A. Papadopoulos and A.D. Dimarogonas, Coupled vibration of cracked shafts, ASME J. Vibration and Acoustics 114 (1992), 461-467.

[20] P.F. Rizos, N. Aspragathos and A.D. Dimarogonas, Identification of crack location and magnitude in a cantilevel beam from the vibration modes, J. Sound and Vibration 138 (1990), 381388 .

[21] M.H.H. Shen and C. Pierre, Free vibrations of beams with a single-edge crack, J. Sound and Vibration 170 (1994), 237259.

[22] W.J. Thomson, Vibration of slender bars with discontinuities in stiffness, J. Applied Mechanics 17 (1943), 203-207.

[23] D. Wendtland, Aenderung der biegeeigenfrequenzen einer idealisierten Schaufel durch Risse, Ph.D. thesis, University of Karlsruhe, 1972.

[24] M.C. Wu and S.C. Huang, In-plane vibration and crack detection of a rotating shaft-disk containing a transverse crack, ASME J. Vibration and Acoustics 120 (1998), 551-556. 

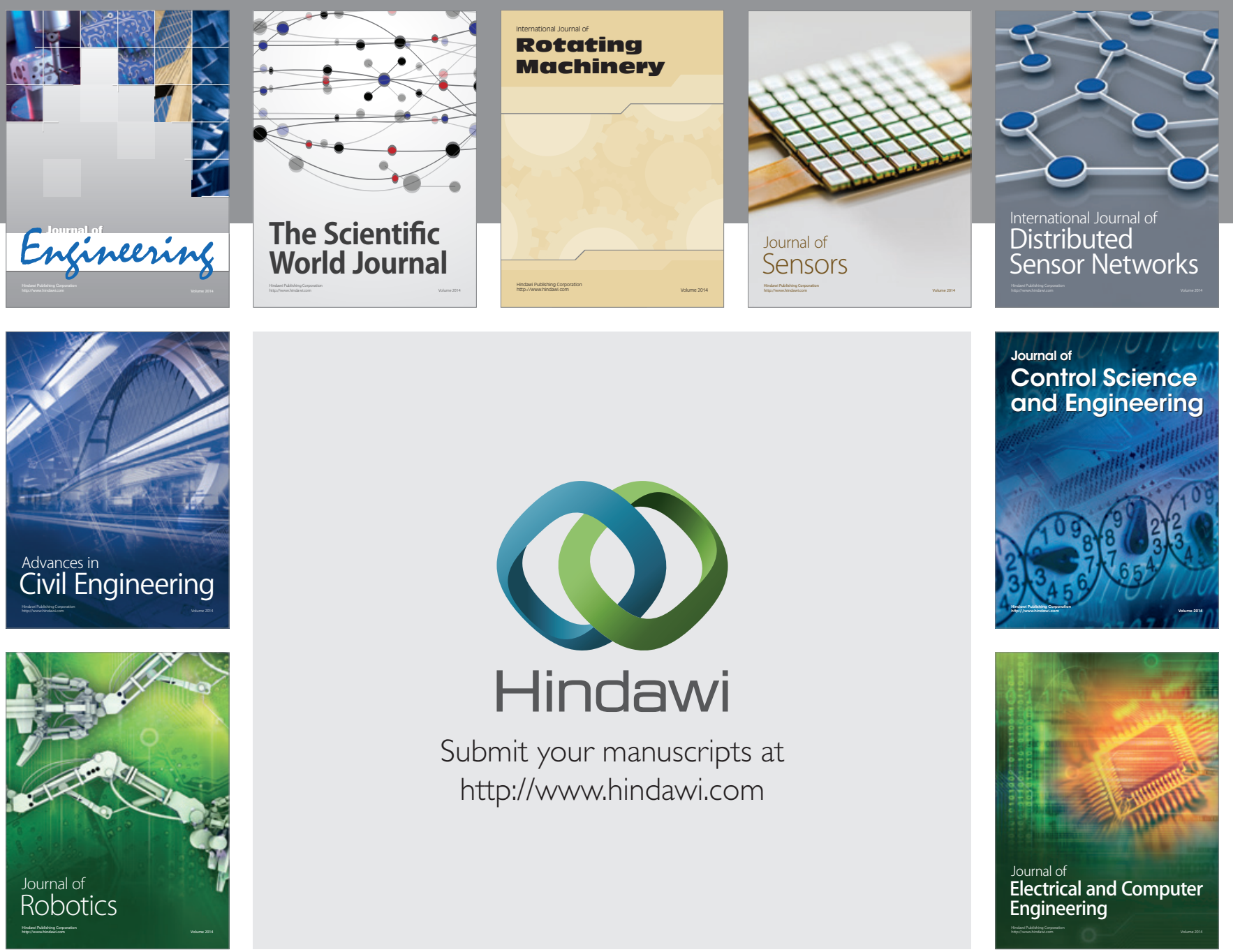

Submit your manuscripts at

http://www.hindawi.com
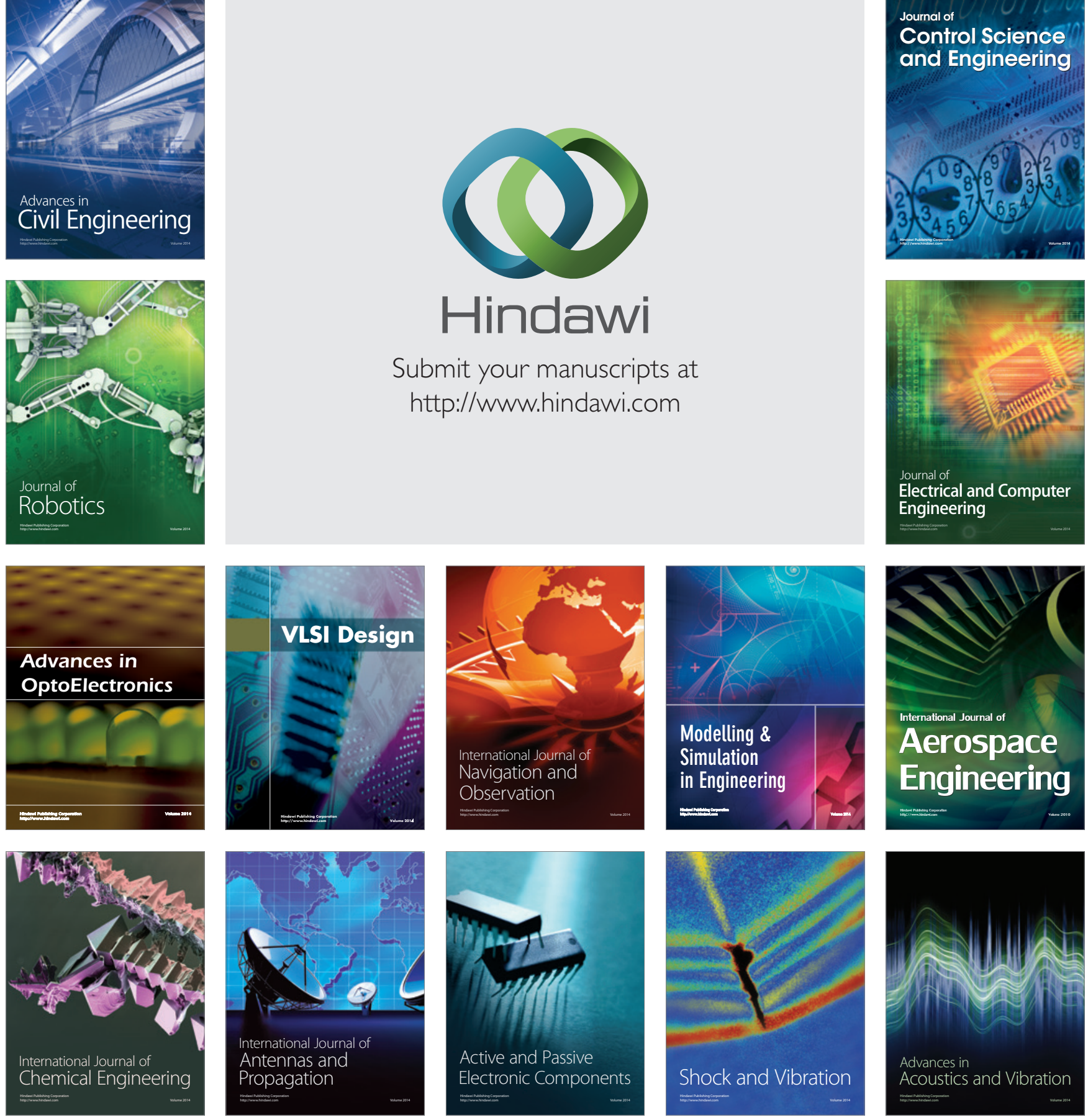